\title{
Recent Updates on Subcortical Ischemic Vascular Dementia
}

\author{
Jee Hoon Roh, ${ }^{\mathrm{a}, \mathrm{b}}$ Jae-Hong Lee ${ }^{\mathrm{a}}$ \\ ${ }^{a}$ Department of Neurology, Asan Medical Center, University of Ulsan College of Medicine, Seoul, Korea \\ ${ }^{b}$ Department of Anatomy and Cell Biology, Cell Dysfunction Research Center (CDRC), University of Ulsan College of Medicine, Seoul, Korea
}

Vascular dementia $(\mathrm{VaD})$ is a history-laden disease entity that dates back to the 19th century when arteriosclerotic brain atrophy due to hardening of the arteries was perceived as the major cause of senile dementia. Its existence had been overshadowed by the emergence of Alzheimer's disease (AD) in the past century and research on $A D$ dominated the field of dementia. Interest in $\mathrm{VaD}$ has been revived in recent years as vascular lesions have been shown to make great contributions to the development of dementia, particularly in the elderly. $\mathrm{VaD}$ has now evolved into the concept of vascular cognitive impairment (VCl), which encompasses not only $\mathrm{VaD}$ but also $\mathrm{AD}$ with cerebrovascular disorder and $\mathrm{VCl}$ with no dementia. The concept of $\mathrm{VCl}$ is intended to maximize the therapeutic potential in dementia management because the vascular component may be amenable to therapeutic intervention particularly in the early stages of cognitive impairment. Subcortical ischemic vascular dementia (SIVD) is pathologically driven by severe stenosis and the occlusion of small vessels that culminate into white matter ischemia and multiple lacunar infarctions in the subcortical structures. The relatively slow progression of symptoms and clinical manifestations associated with cholinergic deficits often make the differentiation of SIVD from AD difficult. The recent development of in vivo amyloid imaging enabled further pathological breakdown of SIVD into pure SIVD and mixed dementia with subcortical ischemia based on the absence or existence of amyloid pathology in the brain. In this article, the authors reviewed the emerging concepts of $\mathrm{VaD} / \mathrm{NCl}$ and the clinical manifestations, biomarkers, treatments, and preclinical models of SIVD based on the pathophysiologic mechanisms of the disease.

Keywords Vascular dementia; Vascular cognitive impairment; Subcortical ischemic vascular dementia; Alzheimer's disease

\author{
Correspondence: Jae-Hong Lee \\ Department of Neurology, Asan Medical \\ Center, University of Ulsan College of \\ Medicine, 88 Olympic-ro 43-gil, \\ Songpa-gu, Seoul 138-736, Korea \\ Tel: +82-2-3010-3446 \\ Fax: +82-2-474-4691 \\ E-mail:jhlee@amc.seoul.kr \\ Received: July 31, 2013 \\ Revised: September 9, 2013 \\ Accepted: October 28, 2013 \\ This research was supported by the Basic \\ Science Research Program through the \\ National Research Foundation of Korea \\ (NRF) funded by the Ministry of Science, \\ ICT \& Future Planning \\ (2013R1A1A1012925), NRF MRC grant \\ funded by the Korea government (MSIP. \\ 2008-0062286), the KIST Institutional \\ Program (2E24242-13-110), a grant \\ (2013-590) from the Asan Institute for Life \\ Sciences (JHR), and a grant from CJ Pharma \\ (2009-0497) (JL), Seoul, Korea.
}

The authors have no financial conflicts of interest.

\section{Introduction}

Vascular dementia $(\mathrm{VaD})$ is caused by cerebrovascular disease that directly or indirectly damages the brain structures associated with cognitive functioning. Historically, the concept of VaD has been described since the 19th century when arteriosclerotic brain atrophy due to hardening of the arteries was per- ceived as the major cause of senile dementia. ${ }^{1,2}$ The importance of $\mathrm{VaD}$ was overshadowed in the past century by the emergence of Alzheimer's disease (AD), which has turned out to be the most common cause of dementia. Interest in $\mathrm{VaD}$ has been revived in recent years because vascular lesions have been continuously noted to be substantial contributors to the development of dementia by themselves or by their synergetic effects on the 
pathogenesis associated with $\mathrm{AD},{ }^{3,4}$ With accumulating evidences suggesting the role of vascular burden on cognitive functions, $\mathrm{VaD}$ has now evolved into the concept of vascular cognitive impairment (VCI), which encompasses not only $\mathrm{VaD}$ but also $\mathrm{AD}$ with cerebrovascular disorder $(\mathrm{AD}$ with $\mathrm{CVD}$ or mixed dementia) and VCI with no dementia (VCIND). Thus, VCI is a more broad term that comprises all the states of cognitive impairment associated with CVD. ${ }^{3-5}$ Around one-third of patients with $\mathrm{AD}$ demonstrate evidences of vascular pathology, ${ }^{6-8}$ while up to two-thirds of patients with CVD develop at least some degree of $\mathrm{AD}$ pathology in the brain. ${ }^{9,10}$ Subcortical ischemic vascular dementia (SIVD) is one of the VaDs which can have both vascular and $\mathrm{AD}$ associated pathology in the brain. SIVD is pathologically driven by stenosis and occlusion of small vessels, resulting in white matter ischemia and multiple lacunar infarctions in subcortical structures. SIVD is of particular interest as the relatively slow progression of symptoms and clinical manifestations of the disease often make the differentiation of it from $\mathrm{AD}$ difficult. In this article, the authors reviewed the emerging concepts of $\mathrm{VaD} / \mathrm{VCI}$ and clinical manifestations, biomarkers, treatments, and preclinical models of SIVD based on the pathophysiologic mechanisms of the disease.

\section{Emerging concepts in VaD and VCI}

$\mathrm{VaD}$ can be classified into the following: 1) multi-infarction dementia, 2) strategic infraction dementia, 3) hemorrhagic dementia, 4) mixed dementia, 5) SIVD, and 6) other forms of vascular dementia. ${ }^{5,11}$ The first three types of VaDs develop relatively suddenly because of acute cerebrovascular diseases and they demonstrate specific cortical or subcortical symptoms depending on the stroke-affected regions. The stepwise progression, fluctuation of symptoms, and focal neurological signs are suggestive of acutely developing $\mathrm{VaD}$. However, the cognitive impairments associated with SIVD often show an insidious onset and progressive decline; thus, mimicking the course of $\mathrm{AD} .{ }^{12}$ Motor symptoms are often not obvious and go unnoticed in this setting, further confusing clinicians. It is suggested that the $\mathrm{AD}$ pathologies are the leading cause of dementia and this can take precedence over the status of cerebral ischemia. ${ }^{12-15} \mathrm{~A}$ recent amyloid imaging study, however, has indicated that there exists a pure SIVD, in which significant subcortical white matter ischemic changes are present with no evidence of amyloid plaque deposition in the brain (Figure 1) ${ }^{16}$ Moreover, pure SIVD is more prevalent than previously thought. This condition is distinctive from
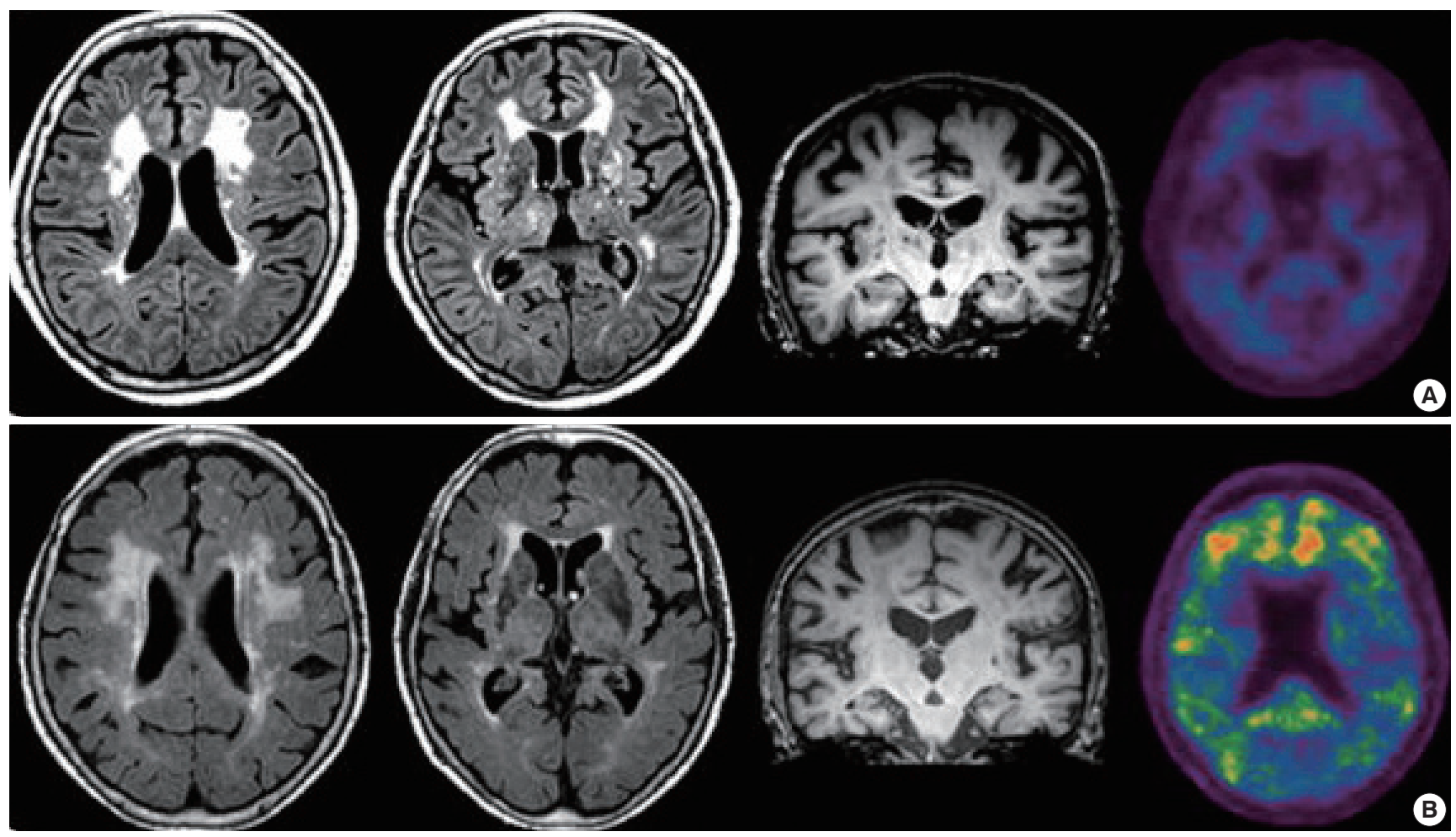

Figure 1. Representative imaging scans of patients with pure subcortical ischemic vascular dementia (PiB-PET negative) or mixed dementia (PiB-PET positive). The first two axial fluid-attenuated inversion recovery images represent a similar degree of white matter hyperintensities of representative subjects in the PiB-negative (A) and in the PiB-positive (B) group. The T1-coronal images in the third column demonstrate a similar degree of generalized cortical atrophy, including medial temporal atrophy, in both the PiB-negative (A) and PiB-positive (B) groups. The PiB-PET scans in the fourth column demonstrate PiB-negativity and PiB-positivity scans, with the red color representing more intracranial PiB retentions. Abbreviations: PiB, 11C-Pittsburgh compound B; PET, positron emission tomography. Modified from Neurology 77(1): 18-25, 2011. 
$\mathrm{AD}$ or mixed dementia, which demonstrate fibrillar forms of amyloid deposition in the brain. ${ }^{16,17}$ Finally, other forms of $\mathrm{VaD}$ include dementia that is caused by heterogeneous etiologies (e.g., vasculitis, cerebral amyloid angiopathy, and hereditary diseases, such as cerebral autosomal dominant arteriopathy with subcortical infarcts and leukoencephalopathy [CADASIL]). ${ }^{18-20}$

There is growing consensus on the concept of mild cognitive impairment $(\mathrm{MCI})$ due to $\mathrm{AD}$ or very mild $\mathrm{AD}$. It is now believed to be early phases of the pernicious accumulation of amyloid beta $(A \beta)$ plaques and neurofibrillary tangles in the brain, with diverse ranges of severity in clinical symptoms. ${ }^{21,22}$ In contrast, the concept of VCI emphasizes the treatable and preventable components of dementia, including the vascular burden in the brain. As such, the concept of VCI has evolved to amplify the potential treatment of vascular factors in order to improve the cognitive impairments because some vascular components are modifiable to prevent additional cerebrovascular deterioration and to ameliorate the current or ongoing cognitive impairments. Even after cognitive impairment sets in, the modulation of vascular risk factors could provide secondary prevention and improvement of the cognitive deficits. ${ }^{5,11,13}$

In line with the emerging concept of the VCI, cerebral smallvessel disease has drawn lots of interest in the field of $\mathrm{AD}$. The prevalence and influence of cerebral small-vessel disease are considered to be significant in dementia, although underestimated so far. ${ }^{23}$ Evidence of small vessel disease found on magnetic resonance images (MRI; e.g., white matter hyperintensities or lacunar infarctions) can be observed in $20 \%-40 \%$ of community-dwelling older persons ${ }^{12}$ and the prevalence of cognitive impairment in association with small vessel disease has been estimated to be as high as $36 \%-67 \%{ }^{13,24}$ In addition, recent studies represent that $\mathrm{AD}$ also increased the incidence of stroke. ${ }^{14,15}$ Thus, it is worthwhile to compare SIVD to $\mathrm{AD}$ in terms of the pathophysiological mechanisms, clinical manifestations, biomarkers, treatment options, and preclinical models.

\section{Pathophysiology of SIVD}

SIVD is mainly caused by widespread ischemic changes in the white matter or multiple lacunar infarctions in subcortical structures. ${ }^{13,23,25-28}$ Predominant arteriosclerotic leukoencephalopathy in the white matter is classically referred to as Binswanger's disease. This type of ischemic change in the brain is formed by widespread incomplete infarctions or hypoperfusion of the white matter due to critical stenosis in the cortical medullary branches. Pathologically, the white matter lesions demonstrate degeneration of the myelin sheath and axons and degradation of the oligodendrocytes without profound infarction or cystic necrosis. On the other hand, the lacunar infarctions (état lacunaire) are caused by perforating arteriolar occlusions in subcortical structures, including the thalamus, basal ganglia, and internal and external capsules. The possible mechanisms underlying the steno-occlusion of small vessels include increased resistance to blood flow, decreased autoregulation, disruption of the bloodbrain barrier (BBB), dysfunction of the endothelium, and dilatation of the perivascular spaces. ${ }^{12,23,27}$ The ultimate disruption of the prefronto-subcortical circuits and thalamocortical circuits by the accumulation of white matter ischemic lesions and lacunar infractions is thought to be an underlying mechanism that result in the cognitive impairments in SIVD. In mixed dementia, which has both the vascular and $\mathrm{AD}$ pathologies in the brain, these alterations in cerebrovascular circulation have been described to contribute to the development of dementia by themselves or by a synergistic interaction with $\mathrm{AD}$ pathology in the brain. ${ }^{29}$ However, the concomitant pathology of $\mathrm{AD}$, including cholinergic deficits and the pathologic accumulations of $A \beta$ plaques and neurofibrillary tangles, has also been found in patients with alleged SIVD, which is also believed to accelerate cerebral ischemia in the brain. ${ }^{16}$

\section{Clinical manifestations of SIVD and its associations with structural and functional brain images}

Along with cognitive impairments, the stepwise development of neurological deficits is not uncommon in patients with SIVD. These deficits include hemiparesis, dysphagia, dysarthria, pseudobulbar palsy, emotional incontinence, urinary incontinence, and parkinsonian features, such as short-stepped gait. Abnormal behaviors, including disinhibition and akinetic mutism, can also develop during the course of SIVD, if the prefronto-subcortical circuits are interrupted directly or indirectly. ${ }^{30}$ Because behavioral changes usually develop later in the course of $\mathrm{AD}$, the early appearance of behavioral or psychological symptoms can suggest the existence of SIVD, particularly when they present in stepwise patterns. Neuropsychological tests can reveal impairments in attention, execution, set-shifting performances, and verbal fluency from the early phases of SIVD. However, patients with $\mathrm{AD}$ demonstrate more difficulties with memory, naming, and visuospatial functions from the beginning of the symptomatic phases. A recent amyloid positron emission tomography (PET) imaging study has shown that patients with $\mathrm{AD}$ having fibrillar forms of $A \beta$ in the brain demonstrate more difficulties in verbal/visual memory-associated tasks, while those with pure SIVD without amyloid pathology in the brain exhibit more trouble during executive functions, such as phonemic fluency of the 
Table 1. Cognitive functions in patients with Subcortical Ischemic Vascular Dementia (SIVD) and Alzheimer's Disease (AD)

\begin{tabular}{lcc}
\hline Cognitive domains & SIVD & AD \\
\hline Attention & $\downarrow \downarrow$ & $\downarrow$ \\
Language and related function & $\downarrow \downarrow$ (fluency) & $\downarrow \downarrow$ (naming) \\
Visuospatial function & $\downarrow$ & $\downarrow \downarrow$ \\
Memory (verbal and visual) & $\downarrow \downarrow$ & $\downarrow \downarrow \downarrow$ \\
Frontal/Executive & $\downarrow \downarrow \downarrow$ & $\downarrow \downarrow$ \\
\hline
\end{tabular}

Controlled Oral Word Association Test and the Stroop color test (Table 1). ${ }^{17,31-33}$ Associations between the amount of white matter pathology and impairments in daily living activities have also been described. ${ }^{34}$

Beyond the signal changes visible on MRI, recent methodological advances have enabled further image-based analyses to investigate the integrity of normal-appearing white matter tracts and the mechanisms that underlie cognitive dysfunctions associated with white matter changes. Previous studies on patients with CADASIL described an increased mean diffusivity and decreased diffusion anisotropy in the areas of white matter hyperintensities, which suggests the disruption of the white matter tracts in SIVD. ${ }^{35,36}$ Although the interpretation is limited due to the small numbers of participants, previous studies on patients with SIVD reported perfusion defects in the bilateral pulvinar nuclei of the thalamus, ${ }^{37}$ caudate nucleus, and diverse cortical areas, including the cingulate, superior temporal, subcallosal gyri, ${ }^{38}$ and frontal lobes. ${ }^{39}$ These findings support the notion of frontosubcortical circuit disruption in patients with SIVD. In addition to structural imaging biomarkers, PET images obtained with amyloid tracers can also be very useful for demonstrating the underlying pathology of SIVD. Although recent guidelines from the Amyloid Imaging Task Force convened by the Alzheimer's Association and the Society of Nuclear Medicine and Molecular Imaging do not recommend using amyloid PET imaging as a routine test for the diagnosis of $\mathrm{AD},{ }^{40,41}$ amyloid PET imaging can be clinically useful for differentiating pure SIVD and mixed dementia, which would sometime be tough to distinguish and would pose diagnostic dilemmas. A new criterion that could help discriminate pure SIVD from mixed dementia is under way using a combination of clinical and MRI findings based on data from amyloid PET imaging.

\section{Preclinical models of SIVD}

Two different models of SIVD have been described in the literature. One is a mechanical model, in which microcoils are applied to the bilateral common carotid arteries to induce continuous hypoperfusion and ischemia in the white matter without apparent gray matter changes. ${ }^{42,43}$ This mouse model has demonstrated decreased hippocampal volume and impaired working memory deficits that are possibly due to damaged frontosubcortical circuits. Subsequent demyelination of the white matter tracks is typical in this model, which can mimic the demyelinating pathology of the SIVD. However, the lack of methods that can affect the actual small vessels is a fundamental limitation of this model, even though the manipulation of the carotid artery does mimic the pathological findings of SIVD. The other model of SIVD is a transgenic mouse model of CADASIL that expresses Notch3 mutations at R90C..$^{44}$ As CADASIL shares many pathophysiological and clinical manifestations with SIVD, research on SIVD and CADASIL is closely associated. Mouse models of CADASIL also develop similar cerebrovascular changes in the brain as in humans. ${ }^{45,46}$ However, the limited volume of white matter in rodents is a drawback and mouse models of CADASIL do not demonstrate widespread white matter changes in the brain. Recent advances in technology will make the modulation of the small vessels easier and more reliable. If new mouse models of SIVD can be introduced, it will allow better understanding of the direct pathological burden of the CVD on cognition, which can be separated from other cognitive impairments driven by neurodegenerative etiologies, such as accumulation of $A \beta$ plaques or neurofibrillary tangles.

\section{SIVD Biomarkers}

Various results have been described in biomarker studies on patients with $\mathrm{VaD} / \mathrm{VCI}$. A recent longitudinal study has compared the baseline cerebrospinal fluid (CSF) biomarkers of four different groups: a $\mathrm{MCI}$ group that finally progressed to $\mathrm{AD}$ (MCI-AD), mixed dementia (MCI-MD), or SIVD (MCI-SIVD), and a MCI group that remained stable as MCI (MCI-MCI). ${ }^{47}$ The results from CSF $A \beta$ and tau showed that the levels of $A \beta$ were higher and the levels of total tau and the phosphorylated forms of tau ( $\mathrm{p}$-tau) were lower in the MCI-SIVD group than in the MCI-AD group. Interestingly, the levels of CSF biomarkers obtained in the MCI-MD group were in between the levels of the MCI-AD group and the MCI-SIVD group. The CSF levels obtained from the MCI-SIVD group were closer to the levels of the MCI-MCI group and the normal control group. The intermediate CSF levels of $A \beta$ and tau in the SIVD group compared to the mixed dementia/AD and normal controls indicate that SIVD possibly shares a common pathophysiology with $\mathrm{AD}$. When patients with $\mathrm{VaD}$ and $\mathrm{AD}$ are compared, the levels of CSF $\mathrm{A} \beta$ had a tendency to be lower and CSF tau had a tendency to be higher in patients with $\mathrm{AD}$ than in patients with $\mathrm{VaD}$, but substantial overlaps existed between the groups. ${ }^{48,49}$ On the other 
hand, another study that compared the levels of CSF A $\beta$ and tau among non-demented elderly persons who had mild, moderate or severe white matter hyperintensities, demonstrated no differences in the levels of CSF A $\beta$ and tau among the groups. ${ }^{50}$

In addition, other markers associated with BBB breakdown have been introduced as alternative biomarkers associated with SIVD. A compromised BBB can be a source of albumin leakage, which can increase protein concentration in the CSF as it crosses the $\mathrm{BBB}$ with less resistance. With this pathological evidence of BBB disruption in patients with SIVD, the increased levels of albumin have been described as supportive evidence of SIVD. ${ }^{51-53}$ Metalloproteinases are markers of neuroinflammation as they attack the basal lamina and tight junctions in blood vessels, in addition to the disruption of myelin. ${ }^{54}$ In comparison with $\mathrm{AD}$, the levels of metalloproteinases-9 in the CSF were increased in patients with VCI. ${ }^{55,56}$ Another marker of white matter disruption is neurofilament, which functions as a cytoskeleton in large myelinated axons. ${ }^{57}$ The concentrations of the neurofilament light subunit were reported to be higher in subjects with SIVD. ${ }^{47,58}$ Similar findings were noted in nondemented subjects with severe white matter lesions. ${ }^{50}$ As briefly described in the previous section, advanced imaging technologies can also serve as biomarkers, which enable further discrimination of the underlying pathophysiologies of SIVD.

\section{Treatment of SIVD}

SIVD treatment strategies include both slowing or mitigating underlying small vessel disease progression and the improvement of clinical symptoms. Microinfarction pathology stands out as a significant and independent factor that contributes to brain atrophy and cognitive impairment. ${ }^{26,59,60}$ Therefore, as a secondary prevention of stroke by modulating small vessel disease, antiplatelet agents can be administered. However, the effectiveness of antiplatelet therapy for the primary prevention of $\mathrm{VCI}$ has not yet been established. ${ }^{61} \mathrm{~A}$ calcium-channel blocker, nimodipine, has been introduced as a SIVD treatment because of its vasodilation effects. Following potential success in early trials of nimodipine in patients with $\mathrm{VaD}$, an intention-to-treat trial was conducted with a total number of 230 patients. In that study, treatment with nimodipine did not reach the primary endpoint defined by the researchers, although the treatment group demonstrated improved lexical production and better results on the Mini-Mental State Examination and Global Deterioration Scale. ${ }^{62,63}$ In addition, cholinesterase inhibitors have been introduced to manage cognitive symptoms. Cholinergic deficits in SIVD were described based on findings that the basal forebrain cholinergic nuclei are supplied by perforating arterioles and that the CA1 sector of the hippocampus is vulnerable to ischemic insults. ${ }^{64,65}$ Patients with CADASIL have also dem-

\section{Vascular etiologies}

Cerebrovascular disorders Vascular risk factors

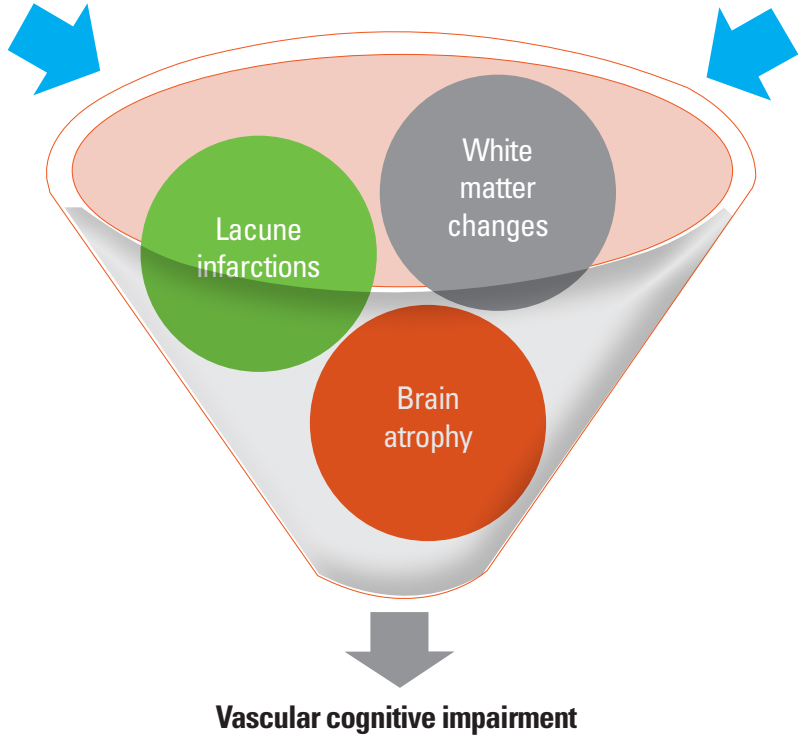

Neurodegenerative etiologies

Alzheimer's disease pathology Aging

Figure 2. Pathophysiology of vascular cognitive impairment. Both vascular and neurodegenerative processes culminate into the development of vascular cognitive impairment. The intervention of modifiable vascular etiologies potentially mitigates and slows the cognitive impairments in patients with vascular cognitive impairments. The progression of Alzheimer's pathologies in the brain is not amenable to current therapeutic interventions. Images modified from $\mathrm{J}$ Korean Neurol Assoc 21(5): 445-454, 2003. 
onstrated deficits in cholinergic fibers. Accordingly, cholinesterase inhibitors have been introduced in the treatment of $\mathrm{VaD}$ for the cognitive impairments associated with cholinergic deficits but not so much for other cognitive dysfunctions. ${ }^{66} \mathrm{~A}$ recent randomized controlled drug trial on patients with CADASIL has also reported the potential beneficial effects of cholinesterase inhibitors on cognitive functions. ${ }^{67}$ In another study of CADASIL, treatment with the cholinesterase inhibitor, donepezil, did not improve the primary endpoints measured by the vascular Alzheimer's disease assessment scale-cognitive subscale (VADAS-Cog) scores. However, a subgroup analysis indicated improvements in executive functions in the treatment group. ${ }^{67}$ Antidepressants, such as selective serotonin reuptake inhibitors, can also be used for the management of depression and atypical neuroleptics can be selectively used for behavioral changes, including agitation and aggression. Recently, modification of cardiovascular risk factors has been recommended for the prevention of $\mathrm{AD}$ and VCI, including the SIVD. The modifiable and preventive risk factors include hypertension, diabetes, and hypercholesterolemia. In addition, modifications of lifestyle, including education, diet, physical activity, alcohol consumption, smoking, obesity, and social support/networking, can also be considered for the management of VCI (Figure 2). ${ }^{61}$

\section{Conclusions}

$\mathrm{VaD}$ is a heterogeneous disease entity that encompasses various conditions. SIVD is a relatively homogeneous vascular dementia that can mimic $\mathrm{AD}$ due to the slow progression of cognitive decline. Detailed medical histories and neuropsychological tests can reveal early impairments in frontal executive symptoms and behavioral or mood changes. Neuroimaging studies and CSF biomarkers can also help in diagnosing and differentiating SIVD from other causes of dementia. Proper and early diagnosis, with careful review of the patients' medical history, neurological findings, structural and/or functional neuroimaging, and information from biomarkers, will enable clinicians to select the proper management techniques for the secondary prevention of stroke and the symptomatic treatment of SIVD. An improved understanding of the pathophysiology of SIVD with diverse experimental models of SIVD as well as the development and validation of new biomarkers will make the diagnosis and treatment of SIVD more reliable and effective. Finally, well-designed longitudinal studies with imaging and biological biomarkers in SIVD and subcortical vascular MCI, such as the Clinical Research Center for Dementia of South Korea (CREDOS) ${ }^{68}$ the Amyloid PET Imaging for Subcortical Vascular Dementia study (AMPETIS), ${ }^{16}$ and the Korean Alzheimer's
Disease Neuroimaging Initiative (K-ADNI), will shed light on efforts to better understand the pathophysiology and prognosis of SIVD.

\section{Future directions}

Data regarding VaD, including SIVD, need to be collected, maintained as a database in a standardized fashion, and shared among researchers to better define and manage dementia. The standardization of vascular lesions in neuropathology and neuroimaging is well under way. More work remains to be done before the concept of SIVD is widely accepted and put actively into clinical practice.

\section{References}

1. Roman GC. A historical review of the concept of vascular dementia: lessons from the past for the future. Alzheimer Dis Assoc Disord 1999;13(Suppl 3):S4-8.

2. Roman GC. On the history of lacunes, etat crible, and the white matter lesions of vascular dementia. Cerebrovasc Dis 2002;13 (Suppl 2):1-6.

3. Hachinski V, Iadecola C, Petersen RC, Breteler MM, Nyenhuis DL, Black SE, et al. National Institute of Neurological Disorders and Stroke-Canadian Stroke Network vascular cognitive impairment harmonization standards. Stroke 2006;37:22202241.

4. Farooq MU, Gorelick PB. Vascular cognitive impairment. Curr Atheroscler Rep 2013;15:330.

5. Pendlebury ST, Rothwell PM. Prevalence, incidence, and factors associated with pre-stroke and post-stroke dementia: a systematic review and meta-analysis. Lancet Neurol 2009;8:10061018 .

6. Knopman DS, Parisi JE, Boeve BF, Cha RH, Apaydin H, Salviati A, et al. Vascular dementia in a population-based autopsy study. Arch Neurol 2003;60:569-575.

7. Neuropathology Group. Medical Research Council Cognitive Function and Aging Study. Pathological correlates of late-onset dementia in a multicentre, community-based population in England and Wales. Neuropathology Group of the Medical Research Council Cognitive Function and Ageing Study (MRC CFAS). Lancet 2001;357:169-175.

8. White L, Petrovitch H, Hardman J, Nelson J, Davis DG, Ross GW, et al. Cerebrovascular pathology and dementia in autopsied Honolulu-Asia Aging Study participants. Ann N Y Acad Sci 2002;977:9-23.

9. Jin YP, Di Legge S, Ostbye T, Feightner JW, Hachinski V. The reciprocal risks of stroke and cognitive impairment in an elder- 
ly population. Alzheimers Dement 2006;2:171-178.

10. Yu KH, Cho SJ, Oh MS, Jung S, Lee JH, Shin JH, et al. Cognitive impairment evaluated with Vascular Cognitive Impairment Harmonization Standards in a multicenter prospective stroke cohort in Korea. Stroke 2013;44:786-788.

11. Pendlebury ST. Stroke-related dementia: rates, risk factors and implications for future research. Maturitas 2009;64:165-171.

12. Chui HC. Subcortical ischemic vascular dementia. Neurol Clin 2007;25:717-740, vi.

13. Roman GC, Erkinjuntti T, Wallin A, Pantoni L, Chui HC. Subcortical ischaemic vascular dementia. Lancet Neurol 2002;1: 426-436.

14. Tolppanen AM, Lavikainen P, Solomon A, Kivipelto M, Soininen $\mathrm{H}$, Hartikainen S. Incidence of stroke in people with $\mathrm{Al}$ zheimer disease: a national register-based approach. Neurology 2013;80:353-358.

15. Chi NF, Chien LN, Ku HL, Hu CJ, Chiou HY. Alzheimer disease and risk of stroke: a population-based cohort study. Neurology 2013;80:705-711.

16. Lee JH, Kim SH, Kim GH, Seo SW, Park HK, Oh SJ, et al. Identification of pure subcortical vascular dementia using 11C-Pittsburgh compound B. Neurology 2011;77:18-25.

17. Yoon CW, Shin JS, Kim HJ, Cho H, Noh Y, Kim GH, et al. Cognitive deficits of pure subcortical vascular dementia vs. Alzheimer disease: PiB-PET-based study. Neurology 2013;80:569-573.

18. Seo SW, Hwa Lee B, Kim EJ, Chin J, Sun Cho Y, Yoon U, et al. Clinical significance of microbleeds in subcortical vascular dementia. Stroke 2007;38:1949-1951.

19. Park JH, Seo SW, Kim C, Kim GH, Noh HJ, Kim ST, et al. Pathogenesis of cerebral microbleeds: in vivo imaging of amyloid and subcortical ischemic small vessel disease in 226 individuals with cognitive impairment. Ann Neurol 2013;73:584-593.

20. Patel B, Lawrence AJ, Chung AW, Rich P, Mackinnon AD, Morris $\mathrm{RG}$, et al. Cerebral microbleeds and cognition in patients with symptomatic small vessel disease. Stroke 2013;44:356361.

21. Morris JC. Revised criteria for mild cognitive impairment may compromise the diagnosis of Alzheimer disease dementia. Arch Neurol 2012;69:700-708.

22. Sperling RA, Aisen PS, Beckett LA, Bennett DA, Craft S, Fagan AM, et al. Toward defining the preclinical stages of Alzheimer's disease: recommendations from the National Institute on Aging-Alzheimer's Association workgroups on diagnostic guidelines for Alzheimer's disease. Alzheimers Dement 2011;7: 280-292.

23. Pantoni L. Cerebral small vessel disease: from pathogenesis and clinical characteristics to therapeutic challenges. Lancet Neurol 2010;9:689-701.
24. Chui H. Dementia due to subcortical ischemic vascular disease. Clin Cornerstone 2001;3:40-51.

25. Arvanitakis Z, Leurgans SE, Barnes LL, Bennett DA, Schneider JA. Microinfarct pathology, dementia, and cognitive systems. Stroke 2011;42:722-727.

26. Vinters HV, Ellis WG, Zarow C, Zaias BW, Jagust WJ, Mack WJ, et al. Neuropathologic substrates of ischemic vascular dementia. J Neuropathol Exp Neurol 2000;59:931-945.

27. Ross R. The pathogenesis of atherosclerosis: a perspective for the 1990s. Nature 1993;362:801-809.

28. Ross R. Atherosclerosis--an inflammatory disease. $N$ Engl J Med 1999;340:115-126.

29. van Norden AG, van Dijk EJ, de Laat KF, Scheltens P, Olderikkert MG, de Leeuw FE. Dementia: alzheimer pathology and vascular factors: from mutually exclusive to interaction. Biochim Biophys Acta 2012;1822:340-349.

30. Choi SH, Kim S, Han SH, Na DL, Kim DK, Cheong HK, et al. Neurologic signs in relation to cognitive function in subcortical ischemic vascular dementia: a CREDOS (Clinical Research Center for Dementia of South Korea) study. Neurol Sci 2012; 33:839-846.

31. Graham NL, Emery T, Hodges JR. Distinctive cognitive profiles in Alzheimer's disease and subcortical vascular dementia. J Neurol Neurosurg Psychiatry 2004;75:61-71.

32. Fuh JL, Wang SJ, Cummings JL. Neuropsychiatric profiles in patients with Alzheimer's disease and vascular dementia. J Neurol Neurosurg Psychiatry 2005;76:1337-1341.

33. Looi JC, Sachdev PS. Differentiation of vascular dementia from AD on neuropsychological tests. Neurology 1999;53:670-678.

34. Moon SY, Na DL, Seo SW, Lee JY, Ku BD, Kim SY, et al. Impact of white matter changes on activities of daily living in mild to moderate dementia. Eur Neurol 2011;65:223-230.

35. Chabriat H, Pappata S, Poupon C, Clark CA, Vahedi K, Poupon $\mathrm{F}$, et al. Clinical severity in CADASIL related to ultrastructural damage in white matter: in vivo study with diffusion tensor MRI. Stroke 1999;30:2637-2643.

36. Matthews PM, Filippini N, Douaud G. Brain structural and functional connectivity and the progression of neuropathology in Alzheimer's disease. J Alzheimers Dis 2013;33(Suppl 1):S163172.

37. Kato H, Yoshikawa T, Oku N, Imaizumi M, Takasawa M, Kimura $\mathrm{Y}$, et al. Statistical parametric analysis of cerebral blood flow in vascular dementia with small-vessel disease using Tc-HMPAO SPECT. Cerebrovasc Dis 2008;26:556-562.

38. Yang DW, Kim BS, Park JK, Kim SY, Kim EN, Sohn HS. Analysis of cerebral blood flow of subcortical vascular dementia with single photon emission computed tomography: adaptation of statistical parametric mapping. J Neurol Sci 2002;203-204:199- 
205.

39. Shyu WC, Lin JC, Shen CC, Hsu YD, Lee CC, Shiah IS, et al. Vascular dementia of Binswanger's type: clinical, neuroradiological and 99mTc-HMPAO SPET study. Eur J Nucl Med 1996; 23:1338-1344.

40. Johnson KA, Minoshima S, Bohnen NI, Donohoe KJ, Foster NL, Herscovitch P, et al. Appropriate use criteria for amyloid PET: a report of the Amyloid Imaging Task Force, the Society of Nuclear Medicine and Molecular Imaging, and the Alzheimer's Association. J Nucl Med 2013;54:476-490.

41. Johnson KA, Minoshima S, Bohnen NI, Donohoe KJ, Foster NL, Herscovitch P, et al. Appropriate use criteria for amyloid PET: a report of the Amyloid Imaging Task Force, the Society of Nuclear Medicine and Molecular Imaging, and the Alzheimer's Association. Alzheimers Dement 2013;9:e-1-16.

42. Nishio K, Ihara M, Yamasaki N, Kalaria RN, Maki T, Fujita Y, et al. A mouse model characterizing features of vascular dementia with hippocampal atrophy. Stroke 2010;41:1278-1284.

43. Shibata M, Yamasaki N, Miyakawa T, Kalaria RN, Fujita Y, Ohtani R, et al. Selective impairment of working memory in a mouse model of chronic cerebral hypoperfusion. Stroke 2007;38:28262832.

44. Lacombe P, Oligo C, Domenga V, Tournier-Lasserve E, Joutel A. Impaired cerebral vasoreactivity in a transgenic mouse model of cerebral autosomal dominant arteriopathy with subcortical infarcts and leukoencephalopathy arteriopathy. Stroke 2005; 36:1053-1058.

45. Ruchoux MM, Domenga V, Brulin P, MaciazekJ, Limol S, Tournier-Lasserve E, et al. Transgenic mice expressing mutant Notch3 develop vascular alterations characteristic of cerebral autosomal dominant arteriopathy with subcortical infarcts and leukoencephalopathy. Am J Pathol 2003;162:329-342.

46. Dubroca C, Lacombe P, Domenga V, Maciazek J, Levy B, Tournier-Lasserve $\mathrm{E}$, et al. Impaired vascular mechanotransduction in a transgenic mouse model of CADASIL arteriopathy. Stroke 2005;36:113-117.

47. Bjerke M, Andreasson U, Rolstad S, Nordlund A, Lind K, Zetterberg $\mathrm{H}$, et al. Subcortical vascular dementia biomarker pattern in mild cognitive impairment. Dement Geriatr Cogn Disord 2009; 28:348-356.

48. Paraskevas GP, Kapaki E, Papageorgiou SG, Kalfakis N, Andreadou E, Zalonis I, et al. CSF biomarker profile and diagnostic value in vascular dementia. Eur J Neurol 2009;16:205-211.

49. Kaerst L, Kuhlmann A, Wedekind D, Stoeck K, Lange P, Zerr I. Cerebrospinal fluid biomarkers in Alzheimer's disease, vascular dementia and ischemic stroke patients: a critical analysis. J Neurol 2013.

50. Jonsson M, Zetterberg H, van Straaten E, Lind K, Syversen S,
Edman A, et al. Cerebrospinal fluid biomarkers of white matter lesions - cross-sectional results from the LADIS study. Eur J Neurol 2010;17:377-382.

51. Skoog I, Wallin A, Fredman P, Hesse C, Aevarsson O, Karlsson I, et al. A population study on blood-brain barrier function in 85-year-olds: relation to Alzheimer's disease and vascular dementia. Neurology 1998;50:966-971.

52. Wallin A, Blennow K, Fredman P, Gottfries CG, Karlsson I, Svennerholm L. Blood brain barrier function in vascular dementia. Acta Neurol Scand 1990;81:318-322.

53. Wallin A, Sjogren M, Edman A, Blennow K, Regland B. Symptoms, vascular risk factors and blood-brain barrier function in relation to CT white-matter changes in dementia. Eur Neurol 2000;44:229-235.

54. Rosenberg GA. Matrix metalloproteinases in neuroinflammation. Glia 2002;39:279-291.

55. Adair JC, Charlie J, Dencoff JE, Kaye JA, Quinn JF, Camicioli RM, et al. Measurement of gelatinase B (MMP-9) in the cerebrospinal fluid of patients with vascular dementia and Alzheimer disease. Stroke 2004;35:e159-162.

56. Rosenberg GA. Matrix metalloproteinases and their multiple roles in neurodegenerative diseases. Lancet Neurol 2009;8:205216.

57. Rosenberg GA, Sullivan N, Esiri MM. White matter damage is associated with matrix metalloproteinases in vascular dementia. Stroke 2001;32:1162-1168.

58. Wallin A, Sjogren M. Cerebrospinal fluid cytoskeleton proteins in patients with subcortical white-matter dementia. Mech Ageing Dev 2001;122:1937-1949.

59. Launer LJ, Hughes TM, White LR. Microinfarcts, brain atrophy, and cognitive function: the Honolulu Asia Aging Study Autopsy Study. Ann Neurol 2011;70:774-780.

60. Schneider JA, Bennett DA. Where vascular meets neurodegenerative disease. Stroke 2010;41:S144-146.

61. Gorelick PB, Scuteri A, Black SE, Decarli C, Greenberg SM, Iadecola $\mathrm{C}$, et al. Vascular contributions to cognitive impairment and dementia: a statement for healthcare professionals from the american heart association/american stroke association. Stroke 2011;42:2672-2713.

62. Pantoni L, Bianchi C, Beneke M, Inzitari D, Wallin A, Erkinjuntti T. The Scandinavian Multi-Infarct Dementia Trial: a double-blind, placebo-controlled trial on nimodipine in multi-infarct dementia. J Neurol Sci 2000;175:116-123.

63. Pantoni L, del Ser T, Soglian AG, Amigoni S, Spadari G, Binelli $D$, et al. Efficacy and safety of nimodipine in subcortical vascular dementia: a randomized placebo-controlled trial. Stroke 2005; 36:619-624.

64. Pantoni L. Treatment of vascular dementia: evidence from tri- 
als with non-cholinergic drugs. J Neurol Sci 2004;226:67-70.

65. Gottfries CG, Blennow K, Karlsson I, Wallin A. The neurochemistry of vascular dementia. Dementia 1994;5:163-167.

66. Erkinjuntti T, Roman G, Gauthier S, Feldman H, Rockwood $K$. Emerging therapies for vascular dementia and vascular cognitive impairment. Stroke 2004;35:1010-1017.

67. Dichgans M, Markus HS, Salloway S, Verkkoniemi A, Moline
M, Wang $Q_{2}$ et al. Donepezil in patients with subcortical vascular cognitive impairment: a randomised double-blind trial in CADASIL. Lancet Neurol 2008;7:310-318.

68. Noh Y, Lee Y, Seo SW, Jeong JH, Choi SH, Back JH, et al. A New Classification System for Ischemia Using a Combination of Deep and Periventricular White Matter Hyperintensities. J Stroke Cerebrovasc Dis 2013. 